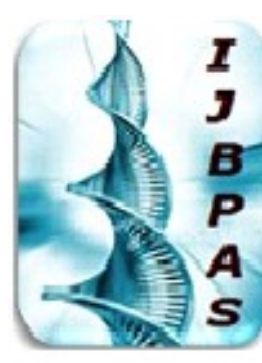

International Journal of Biology, Pharmacy and Allied Sciences (IJBPAS)

'A Bridge Betueen Caboratory and Q ando'

WwW.ibpas.com

\title{
MICROSPHERES FOR CONTROLLED RELEASE DRUG DELIVERY: AN \\ OVERVIEW ON PREPARATION METHODS AND CHARACTERISATION
}

\author{
HANEESHA SK ${ }^{*}$, VENKATA RM, RAO NR \\ Department of Pharmaceutics, Chalapathi Institute of Pharmaceutical Sciences, Chalapathi \\ Nagar, Lam, Guntur-522034
}

"Corresponding Author: SK. Haneesha: E Mail: haneesha6367@gmail.com; Phone no: 6305102651; Fax no: 0863-2524123

Received $15^{\text {th }}$ Oct. 2019; Revised $10^{\text {th }}$ Nov. 2019; Accepted $19^{\text {th }}$ Dec. 2019; Available online $1^{\text {st }}$ April 2020

https://doi.org/10.31032/IJBPAS/2020/9.4.4995

\begin{abstract}
Microspheres are the multiparticulate drug delivery systems which are manufactured to acquire prolonged or controlled drug delivery, which can enhance bioavailability, stability and also the drug can be delivered at a predetermined rate. Some of the difficulties of conventional therapy can be controlled by an effectively designed controlled drug delivery system and the therapeutic efficacy of a given drug is enhanced. Vast attention has been attained by this microspheric drug delivery system because of its prolonged release and also its wide range of application in drug targeting to the particular site in order to maximize the concentration of drug in a particular tissue or organ of the body that surpass the drug's therapeutic efficacy, toxicity can be reduced and improves the patient compliance and patient comfort. Various approaches have been developed to deliver a drug to particular site in a sustained controlled release manner. Among them, one of the approaches is utilizing microspheres as drug carriers. Microspheres were used not only for prolonged release, but the anticancer drugs to the tumour also can be targeted. They consist of a drug which is centrally placed and a unique polymeric membrane that is biodegradable in nature is used to enclose it. The present review is anxious about the microspheres as novel drug delivery system. The main aim of the present study is to review various features of microspheres, methods used for preparation and various applications as targeted or controlled drug delivery system.
\end{abstract}

Keywords: Microspheres, conventional, targeted, applications, controlled release 


\section{INTRODUCTION}

Oral drug delivery route is perhaps the preferred way to take medicines. Though, their short circulation half- life and limited absorption through a specified intestine segment restricts several other drugs' therapeutic effects [1]. Currently, no drug delivery system is ideally conductive to achieving all the ambitious goals, but genuine efforts have been made to accomplish them via novel drug delivery approaches. A number of new drug delivery technologies have developed that include different paths of implementation to ensure managed and targeted delivery of drugs[3]. The focused drug delivery method was designed to try to concentrate the drug in the tissues of interest while increasing the overall concentration of the drug in the other tissues. The drug is therefore placed on the target site. Therefore, the drug does not affect the surrounding tissues [2]. Microspheres were tiny spherical bodies with just a diameter of 1 to 1000 in the $\mu \mathrm{m}$ range [4]. Microparticles from different natural and synthetic components can be produced. It is possible to alter the drug behaviour in vivo by mixing the drug with a carrier molecule. The carrier's activity drastically alters clearance kinetics, tissue distribution, metabolism i.e., kinetics and cellular interaction of the drug. Using these improvements in the action of pharmacodynamics will result in increased therapeutic output. A wide range of materials namely immunoglobulin serum proteins, liposomes, microspheres, microcapsules, nanoparticles and even cells like erythrocytes were also used as drug carriers [5]. Oral microspheres were used to support the release of drugs and to reducing or eliminating discomfort of the gastrointestinal tract. Moreover, multiparticulate delivery systems in the gastrointestinal tract distributed more evenly. This tends to result in a much more reproducible uptake of the drug and lessens local irritation better than single unit dosage forms along with no disintegrating polymer tablets. It is also possible to avoid excessive intestinal accumulation of the polymeric content which can arise with matrix tablets during chronic dosing [6]. In 1997, microspheres were first designed for the drug's continuous action. Microparticles have since proven to also be good fits for continued and controlled release of drugs and became an option to conventional or immediate release compositions. Such particles also provide the active drug ingredients that are pharmacologically active but are hard to deliver due to the low water solubility. In such kinds of drugs, obtaining the necessary therapeutic concentrations of the 
medication throughout the blood stream is difficult, allowing higher levels of $\mathrm{C}_{\max }, \mathrm{t}_{\max }$ and area under curve to be achieved. Microspheres based formulations are capable of releasing a steady amount of drug throughout the blood or targeting drugs to specific body sites. While establishing the drug delivery system, some of the key points to be taken into account are the type of carrier used, the route of administration, the drug delivery target and the strategy to improve the therapeutic effectiveness of the drug. These were all the factors that can be minimize the adverse effects of the active pharmaceutical entity [9].

\section{TYPES OF MICROSPHERES}

\section{Bioadhesive micropsheres:}

Such microspheres show an extended period of residence at the application site due to bioadhesivity which include close contact with that of the site of absorption and ensure continuous release of drugs [15].

\section{Magnetic microspheres:}

These ferromagnetic microspheres are trapped in micro vessels because of their smaller size $(<4 \mu \mathrm{m})$ and drawn by magnetic field into the neighbouring tissues [17].

\section{Floating microspheres:}

Floating microspheres are always the microparticles whose mass is smaller than that of the gastrointestinal fluids and therefore appears buoyant inside the stomach without impacting the pace of gastric emptying. The medication is emitted gradually at the optimal level when the microspheres are floating on gastric component and improves gastric presence and plasma concentration variations [20]. It also reduces the risk of dose dumping and provides a sustained therapeutic effect. Drug (ketoprofen) is given in this form [21].

\section{Mucoadhesive microspheres:}

Mucoadhesive microspheres with a diameter of $1-1000 \mathrm{~mm}$ and comprising of study of a mucoadhesive resin or with an exterior surface and the combination of mucoadhesive properties with microspheres provide additional benefits such as effective uptake and improved bioavailability of the medications because of the increased surface-to-volume ratio, much more direct interaction with that of the mucus membrane. Mucoadhesive microspheres can also be modified to bind to every mucosal tissue, even those located in the eye, nasal cavity, urinary and gastrointestinal tract, thereby providing both local and systematically regulated drug release possibilities [24].

\section{Diagnostic microspheres:}

This can be used to visualize liver cancers. They can also be utilised to separate intestinal loops from many other abdominal 
systems by creating super magnetic iron oxides with Nano particles [25].

\section{Coloured microspheres:}

As per the methodology, specimens were digested using a mixture of enzymatic as well as chemical processes. Aliquots from the microspheres get stuck inside a given species were then measured with an investigator through light microscopy in a hemocytometer. Polystyrene microparticles were coated in one of the five primary colours like white (Blankophor MAR), yellow (Resolin-Brilliant-Gelb 10GN 200\%), violet (Resolin-Rot-violet FBL 200\%) and blue (Resolin-Brilliant-Blan BGLN 200\%) by Bayer AG, Leverkusen, FRG; as well as (Terasil Rot E-BST) from CIBAGEY AG, weher, FRG [28].

\section{Starch microspheres:}

One of their most significant functional characteristics is the potential of polysaccharides to build a matrix structure (gel), even at low doses. The development of the 3D network topology (gelation) is an important way to increase the entire system's chemical equilibrium and mechanical properties. It is understood that a wide variety of altering processes can be added to starches. In contrast, starch is desirable in the field of drug delivery because it enables low-toxicity processes to be developed which are environmentally friendly andvery durable. The cost effectiveness of starch-based products is perhaps other major advantage for use of starch in drug delivery as well as other biomedical uses [28].

\section{Polymer based microspheres:}

According to their possible controlled release features, polymer-based microspheres often gained significant recognition in recent times either through sucking the product constituents from either the polymer or by weakening the polymer matrix. Some organic polymers like proteins, collagen, chitosan and alginate decay by enzymatic action, while artificial polymers including polylactic acid, poly caprolactone, polyglycolic acid and polylactic-co-glycolic acid degrade inside the body hydrolytically [14].

\section{METHODS OF PREPARATION}

\section{Spray drying:}

The polymer should be first dispersed in an effective volatile organic solvent like dichloromethane, acetone. The drug substance in the solid form should then be distributed with high speed homogenization in the polymer. In a flow of warm air, the above dispersion can then be atomized. This atomization contributes to the development of the tiny droplets or the coarse powder wherein the solvent evaporates rapidly going to lead to the production of microparticles within a size range of $1-100 \mu \mathrm{m}$. These microparticles get isolated from the warm air by the use of a cyclone separator where as the liquid 
residue is eliminated by drying the vacuum [1].

\section{Solvent evaporation:}

In this procedure, a drug substance is diluted in chloroform-dissolved polymer and now the corresponding mixture is introduced to the aqueous phase comprising of $0.2 \%$ sodium or pvp like an emulsifier. Above combination was stirred around 500 rpm and hence the drug substance and polymer were converted into some kind of coarse globule that was strengthened by solvent evaporation into solid microspheres and afterwards gathered by filtering and cleaned with demineralised water and stored at room temperature about 24hrs [2].

\section{Single emulsion technique:}

Microparticulate carriers of organic polymers were designed with a single emulsion process that is proteins and carbohydrates. The organic polymers were dispersed in an aqueous media and spread throughout the non-aqueous medium. For the next stage, the dispersed globule is connected either through thermal energy or by chemical cross-connection systems. Through the incorporation of dispersion into earlier heated oil, thermal crosslinking is influenced. Heat denaturation is however not appropriate toward thermo labile drug substances because, when applied and then prone to centrifugation, cleaning and separating the chemical cross linking is unfavourable for unnecessary contact of active compounds to the drug [3].

\section{Double emulsion technique:}

Double emulsion microsphere formulation is perhaps the most appropriate to water soluble materials, peptides, proteins and vaccines and for the production of multiple emulsions or dual emulsion form of $\mathrm{W} / \mathrm{O} / \mathrm{W}$. The aqueous protein mixture can be distributed into a lipid soluble organic continuous process with both inorganic and organic polymers [4]. The active ingredients might be present in this protein solution. The continuous stage comprises in general of the polymer substance which ultimately embodies the protein in the distributed liquid state. The primary emulsion is then homogenized or sonicated before being transferred to the poly vinyl alcohol aqueous solution. It contributes to double emulsion formation. The emulsion is then extracted by solvent evaporation or solvent extraction. The solvent is then extracted [5].

\section{Phase separation coacervation:}

This is the theory of reducing polymer solubility in the organic stage is used to influence the development of polymer rich substances known as co-acervates. This process is used to spread the drug molecules inside a polymer solution and also to introduce an incompatible polymer to the device that separates and absorbs the drug particles in the first polymer. Adding 
non-solvent aids to polymer crystallization.

This method uses butadiene as an inconsistent polymer to build polylactic acid microspheres. A sufficient sped stirrer must be used to eliminate agglomeration by stirring the suspension since the shaped polymerized globules starts to stick to the phase of formation of micropsheres, which form the agglomerates. The process parameters are thus important as they regulate the kinetics of the developed particles, as the equilibrium is not known [5].

\section{Ionic gelation:}

In this process, multivalent cationic or polyanionic is used to compound a hydrophilic polymer to form extremely viscous gel particles. This obtains an opalescent suspension. Then, to receive microspheres, the suspension is centrifuged. Microspheres are dried freezing followed by 24 hour lyophilisation. Owing to electrostatic interactions between positively charged group and negatively charged anion, the resulting microspheres are formed [9].

\section{Super critical fluid technology:}

New SCF preparation technique for pulmonary drug delivery (PDD) microspheres has been developed. SCF procedures were also used for protein and peptide tiny particles. The key problem for protein processing with these methods, however, is related to the protein's exposure to organic solvents, which often leads to a significant decrease in bioactivity and re-agglomeration during storage [8].

\section{Hydroxyl appetite (HAP) microspheres} in sphere morphology:

It was used in the preparation of micropsheres with different morphological features. $\mathrm{O} / \mathrm{W}$ emulsion accompanied by solvent desertion was used to build microspheres. The initial $\mathrm{O} / \mathrm{W}$ emulsion is designed through spreading the organic phase well into the aqueous phase of the surfactant. The untreated step came about as a result of smaller globules surrounded with surfactant particles that held the globules from co-solventing and let them remain single globules. The DCM then dries up gradually while the droplets strengthen into microspheres [30].

\section{ADVANTAGES OF MICROSPHERES}

a) Boost solubility of the poorly soluble drugs by reducing particle size [15].

b) Enhances patient compliance by reducing the frequency of dosing [16].

c) Improved bioavailability despite the first pass impact, as variations in the drug concentration of plasma are easily prevented, the acceptable drug concentration of plasma is ensured by sustained drug release [16]. 
d) Controlled, efficient and targeted medication supply is provided by microspheres [18].

e) Microspheres lower the risk of dose dumping [18].

f) The smell and the taste are also covered by microspheres [18].

g) Reduce core reactivity to the external environment [18].

\section{LIMITATIONS}

a) The prices of the products and production for controlled release formulations are much higher than those for normal formulations [19].

b) The release rate of the controlled dose will differ from a variety of factors such as diet and intestinal movement [20].

c) Controlled release preparations usually contain a larger drug dose and therefore any lack of quantity of the release properties of the dose type can result in serious toxicity [15].

d) A complete glass of water is required to administer this type of dosage form [16].

e) Not acceptable for drugs with problems of solubility or stability in gastric fluids [16].

\section{CHARACTERIZATION}

TECHNIQUES

Particle size and shape:
The most often employed techniques to observe microparticles are conventional light microscopy and scanning electron microscopy [8].

Fourier transform infrared spectroscopy:

An analysis of the interaction studies between both the drug and the excipients was needed to identify. The research has therefore been performed using the FTIR model of SHIMADZU FTIR 410 [27].

\section{X-ray diffraction:}

This technique will assess variations in the product crystallinity. The study of microparticles and their individual components is carried out. The angle of scanning range is $70-80 \mathrm{c}$. Scan detector primary slit $=1 \mathrm{~mm}$, secondary slit $=0.6 \mathrm{~mm}$ [29].

\section{Thermal analysis:}

Thermal analysis of both the microcapsule and its elements can be achieved with differential scanning calorimeter, thermo gravimetric analysis, and differential thermometric analysis [29].

\section{In-vivo method:}

Different types of microsphere release studies are conducted by means of various appropriate dissolution materials, primarily rotating paddle instruments [8].

\section{Drug entrapment efficiency:}

Using the following equation, the drug entrapment efficiency can be calculated [15]. 
$\%$ Entrapment $=$ Actual content $/$

Theoretical content

\section{Stability studies:}

Through positioning the microspheres in a glass container with screw cap and keeping them to the following circumstances [29]. Room temperature $\left(27 \pm 20^{\circ} \mathrm{C}\right), \quad$ Oven temperature $\left(40 \pm 20^{\circ} \mathrm{C}\right)$, Refrigerator (5$\left.8^{\circ} \mathrm{C}\right)$.

\section{Morphological study:}

Scanning electron microscopy was utilized to perform the morphological research. A drug-loaded microspheres' scanning electron microgram was produced. On the glass stub a small quantity of microspheres were placed. After the sample stub has been positioned in the chamber of scanning electron microscope. The photo of microgram was taken at 5.0KV [22].

\section{CONCLUSION}

Microspheric drug delivery mechanism is perhaps the most popular drug delivery system among researches and scientists due to its benefits of controlled and speed of dissolution. Controlled or targeted microspheres enable the effective transport of drugs to an increased surface area, and this drug delivery mechanism serves as a possible tool to enhance drug bioavailability. In contrast, the drug delivery method for microspheres is a successful medication and also an enticing option for the non-invasive delivery of active peptide and likely protein drug molecules. With both the development of new polymers and improved preparation methods and the convergence of many other approaches in the future, microspheres will have a prominent place in the distribution of new drugs.

\section{ACKNOWLEDGEMENT:}

The author would like to thanks Guide Mr. M. Venkata Ramana for his valuable guidance and inspiration for promoting this work. I would like to thanks Dr. N. Rama Rao for their constant support for writing this article.

\section{REFERENCES:}

[1] Kadam NR, Suvarna V. Microspheres: A Brief Review, Asian J Biomed pharm sci. 2015; 5(47):13-19.

[2] Sarlesh rajput,Preethi agarwal, Ashish pathak, Nikhil shrivasthava, Satyendra sigh Bhagal, Rajendra singh Bhagal. A Review on Microspheres: Methods of Preparation and Evaluation. World. J. Pharm. Sci. 2012; 1(1): 422-438.

[3] Poovi Ganesan, Arul Jasmine Deepa Johnson, Lakshmi S, Arun D. Review on Microsphere. Am. J. Drug. Discov. Dev. 2014; 4(3):153179.

[4] Kataria Sahil, Akanksha M, Sandhu Premjeet, Ajay B, Bhawana Kapoor. Microsphere: A Review. 
Int. J. Res. Pharm. Chem. 2011; 1(4): 1184-1198.

[5] Meghana KS, Krishna Pillai M, Sreedevi G, Sreelakshmi C, Vijaykumar B. Microspheres a Drug Delivery System- A Review. Int. J. Novel Trends Pharm. Sci. 2017; 7(4): 109-118.

[6] Sree Giri Prasad B, Guptha RM, Devanna N, Jayasurya K. Microspheres as Drug Delivery System-A Review. J. Global Trends. Pharm. Sci. 2014; 5(3): 1961-1972.

[7] Chitra Singh, Suresh purohit, Madhu Singh, Pandey BL. Design and Evaluation of Microspheres: A Review. J. Drug Deliv. Res. 2013; 2(2): 18-27.

[8] Dalbanjan Nikita S. Microsphere: A Complete Review. World J. Pharm Pharm Sci. 2018; 7(11): 786-817.

[9] Shwetha Saini, Sandeep Kumar, Manjusha Chowdary, Nitesh, Vikaas Budhwar. Microspheres as Controlled Drug Delivery System: An Updated Review. Int. J. Pharm. Sci. Res. 2018; 9(5): 1760-1768.

[10] Mahale Manisha M, Saudagar RB. Microspheres: A Review. J. Drug Deliv. Ther. 2019; 9(3): 854-856.

[11] Alagasundaram M, Madhu sudhana Chetty C, Umashankari K, Venkata Badarinath A,
Lavanaya C, Ramkanth S. Microspheres As a Novel Drug Delivery System-A Review. Int. J. Chem. Tech. Res. 2009; 1(3): 526534.

[12] Vikranth Nikam K, Gudsoorkar VR, Hiremath SN, Dolas RT, Kashid VA. Microspheres-A Novel Drug Delivery System: An Overview. Int. J. Pharm. Chem. Sci. 2012; 1(1): 113-128.

[13] Harsh Bansal, Simar Preet Kaur, Atul Kumar Guptha. Microsphere: Methods Of Preparation and Applications; A Comparitive Study. Int. J. Pharm. Sci. Rev. Res. 2011; 10(1): 69-78.

[14] Kazi M, Zakir Hossain, Uresha Patel, Ifty Ahmed. Development Of Microspheres For Biomedical Applications: A Review. prog biomater. 2015; 4: 1-19.

[15] Anuj Malik. Microsphere: A Novel Drug Delivery System. Original Research Paper. 2017; 6(3): 278-280.

[16] Avinash Kurrey, Preeti Kumaran Suresh, Manju Rwat Singh. Hollow Microspheres As A Drug Carrier: An Overview Of Fabrication and In vivo Characterization Techniques. Chron. Young Sci. 2014; 5(1): 110. 
[17] Krishna Sailaja A, Anusha k. Review On Microspheres As A Drug Carrier. Int. J. Adv. Pharm. 2017; 6(5): 96-102.

[18] Nisha Sharma, Neha Purwar, Prakash Chandra Gupta. Microspheres As Drug Carriers For Controlled Drug Delivery: A Review. Int. J. Pharm. Sci. Res. 2015; 6(11): 4579-4587.

[19] Tarun Virmani, Jyoti Gupta. Pharmaceutical Application of Microspheres: An Approach For The Treatment Of Various Diseases. Int. J. Pharm. Sci. Res. 2017; 8(8): 3252-3260.

[20] Chandrawanshi Mayuri J, Nagoba Shivappa N, Bhalekar Rohini V, Vijayendra Swamy SM. A Review on Microspheres as a Novel Drug Delivery System. Int. J. Pharm. Pharm. Res. 2018; 12(4): 166-185.

[21] Dipak Patil A, Sandi PA, Tadavi, Nilesh P, Salunkhe, Dr. Sunil Pawar P. Review on Microspheres: Methods of Preparation and Evaluation. World J. Pharm. Pharm Sci. 2019; 8(3): 1496-1511.

[22] Ratna Parki MP, Wattamwar MM, Kutmalge MD, Jadhav AN, Chaudhari SP. Mucoadhesive Microsphere-Review. Int. J. Drug. Dev. Res. 2014;6(2): 10-19.
[23] Chun Wong Y, Hani Al-Salami, Crispin Das R. Microsparticles, Microcapsules and Microspheres: A Review of Recent Developmemts and Prospects for Oral Delivery of Insulin. Int. J. Pharm. 2017; 12(36): 1-43.

[24] Divya Rawat, Singh UK, Faizi Muzaffar. Design, Development and Future Application of Microspheres. Pharmatutor. 2019; 11(13): 1-11.

[25] Manoj Kumar Das, Abdul Baquee Ahmed, Dipankar Saha. Microsphere A Drug Delivery Syatem-A Review. Int. J. Curr. Pharm. Res. 2019; 11(4): 34-41.

[26] Neelesh Vardek, Daniel Pack W. Microspheres for Controlled Release Drug Delivery. Expert Opin. Invest. Drugs. 2004; 4(1): $35-51$.

[27] Saravana Kumar K, Jaya Chandra Reddy P, Chandra Sekhar KB. A Review On Microspheres for Novel Drug Delivery System. J. Pharm. Res. 2012; 5(1): 420-424.

[28] Dinesh Kumar, Surender Verma, Kamal. Microspheres: Different Approaches in the Utilization of Microparticulate System-A Review. Int. J. Chem. Stud. 2016; 4(3): 49-53. 
[29] Hire NN, Dr.Derle DV.

Microsphere as Drug Carrier: A

Review. Int. J. Adv. Res. 2014;

2(3): 901-913.

[30] Madan Chopra, Rajesh Asija,

Deepak Sharma, Sangeeta Asija,

Avinash Gupta. A Brief Review

About Microspheres. Int. J. pharm.

Erudition. 2014; 4(3): 9-21. 